\title{
Hs-CRP to albumin ratio predicts mortality of acute myocardial infarction patients with chronic kidney disease undergoing coronary angiography
}

\section{Bo Zuo}

Capital Medical University Affiliated Beijing Friendship Hospital

Jiayu Li

Capital Medical University Affiliated Beijing Friendship Hospital

Hui Chen ( $\nabla$ chenhuibjfh@163.com )

Capital Medical University Affiliated Beijing Friendship Hospital https://orcid.org/0000-0001-98224093

\section{Research}

Keywords: CKD, hs-CRP, HCAR, NT-proBNP, AMI patients, Percutaneous coronary intervention

Posted Date: February 22nd, 2021

DOI: https://doi.org/10.21203/rs.3.rs-197639/v1

License: (a) (1) This work is licensed under a Creative Commons Attribution 4.0 International License. Read Full License 


\section{Abstract \\ Background}

The diagnosis and treatment of acute myocardial infarction (AMI) patients with chronic kidney disease (CKD) is still a challenging problem. The high sensitivity C-reactive protein (hs-CRP) to albumin ratio (HCAR) was proved to be a sensitive biomarker in predicting the prognosis of many diseases. The purpose of this study was to investigate the prognostic value of HCAR in postoperative 2-year mortality of AMI patients with CKD undergoing coronary angiography(CAG).

\section{Methods}

A total of 11933 patients underging CAG were collected in this retrospective study. Finally, $466 \mathrm{AMI}$ patients with CKD undergoing CAG were enrolled. HCAR was calculated by dividing hs-CRP by albumin obtained from blood biochemical examination. Patients were divided into two groups according to the HCAR cutoff value in predicting 2-year mortality by Receiver operating characteristic (ROC) curve analysis. The primary endpoint was all-cause mortality at a 2-year follow-up. The Kaplan-Meier method and Cox regression analyses were used to analyze the survival of patients.

\section{Results}

A significant correlation was found between HCAR and NT-proBNP, LVEF, CK-MB, Tnl and eGFR $(\mathrm{P}<0.05)$. A cut-off value of 0.24 of HCAR predicted 2-year mortality, with a sensitivity of $66.18 \%$ and specificity of $52.21 \%$ (ROC area under the curve: $0.60,95 \% \mathrm{Cl}$ : $0.53-0.68, \mathrm{P}<0.001$ ). A higher HCAR was significantly associated with a higher 2-year mortality rate $(45 / 236(19 \%)$ vs. $23 / 230(10 \%), P=0.006)$. The KaplanMeier curve showed that the group with a higher HCAR had a worse prognosis (log-rank $P<0.001)$. HCAR was an independent risk factor for 2-year mortality (OR: 1.779, 95\% Cl: 1.017-3.112, P = 0.043).

\section{Conclusion}

HCAR might be a potential prognostic indicator of AMI patients with CKD undergoing CAG.

\section{Introduction}

Acute myocardial infarction (AMI), a localized necrosis of the myocardium caused by myocardial ischemia, is still a major contributor to morality worldwide. Early-onset, more severe diseases and complications such as cardiac failure, arrhythmia, cardiogenic and shock are features of AMI.

Percutaneous coronary intervention (PCI) is the main treatment for AMI patients. It can definitely promote the recovery of cardiac function, effectively increase the coronary blood flow and cardiac function reserve by $10 \%-30 \%$, and significantly improve the prognosis and postoperative survival rate of AMI patients[1]. 
However, the treatment of AMI patients with chronic kidney disease (CKD) still poses a challenge. Low glomerular filtration rate, poor renal tubular function and lipid metabolism disorder may lead to complications such as contrast nephropathy and negative outcomes such as sudden cardiac death, arrhythmias, arterial calcification, valve calcification and hemorrhagic stroke, thus the postoperative survival rate and prognosis are less satisfactory $[2,3]$. Literature has shown that as a common inflammatory biomarker in clinics, high sensitivity C-reactive protein (hs-CRP), mainly produced in hepatocytes, is of great value in rehabilitation assessment and associated with atherosclerosis progression and cardiovascular events. Besides, several studies have shown that it is also an independent risk factor for renal function injury[4-6]. In contrast, serum albumin (Alb), a negative phase agent, is preferable to reduce in inflammation. The decrease of Alb usually indicates the aggravation of inflammation, malnutrition and cachexia[7]. Moreover, studies have shown a significant association of low Alb with adverse cardiovascular outcomes in AMI patients, and the level of Alb is also one of the best predictors of mortality in patients with terminal renal failure[8,9]. Studies have shown the CRP to Alb ratio could be used as a sensitive marker of inflammatory process and predicting the prognosis of patients with AMI, critical illness, malignant tumor and severe septicemia[10-13]. Thus, the hs-CRP to Alb ratio (HCAR), calculated by dividing hs-CRP by Alb obtained from clinical blood biochemical examination, may be more valuable in indicating higher residual inflammation with malnutrition status than either alone[14-16].

However, to the knowledge of the authors, no data have been reported on the comparable relationship between the HCAR and the risk of subsequent death in AMI patients with CKD. Therefore, this retrospective study was performed to investigate the relationship between HCAR and postoperative 2-year mortality of AMI patients with CKD undergoing CAG.

\section{Methods}

\section{Study population and data collection}

The present study was a single-center, observational, and retrospective study carried out between January 2013 and August 2019. All the data and follow-up records were derived from Cardiovascular Center of Beijing Friendship Hospital Bank(CCB Bank). A total of 11933 patients underging CAG were collected. This study included 558 patients with CKD, which was defined as an estimated glomerular filtration rate (eGFR) of less than $60 \mathrm{~mL} /$ minute/1.73 m2, as calculated by the MDRD equation, and modified with a Japanese coefficient using baseline serum creatinine. Patients with severe infection or malignancies, lacking complete medical records or lost follow-up were excluded (Fig. 1). A total of 466 patients met the eligibility criteria and were enrolled in the study. Subsequently, the patients were divided into two groups according to the HCAR cutoff value in predicting 2-year mortality by the Receiver operating characteristic (ROC) curve analysis. This study was approved by the ethics committee of Beijing Friendship Hospital, Capital Medical University and written informed consent was obtained from all patients

\section{Laboratory analysis}


The blood samples were collected on the morning of the second day after hospitalization. Blood parameters including serum hs-CRP and albumin levels were detected. The HCAR was determined by dividing serum hs-CRP by serum albumin. Left ventricular ejection fraction (LVEF) was calculated using Simpson's method.

\section{Statistical analysis}

SPSS 21.0 was used for statistical analysis. Normally distributed continuous variables were presented as mean \pm standard deviation, and those with non-normal distribution were presented as median (25th75th), while continuous variables were analyzed using Student's t-test or non-parametric test. Categorical variables were described as absolute values and percentages, and analyzed by the chi-square test. The ROC curve analysis was performed, and Youden index was used to determine the best cut-off value for the HCAR. The Kaplan-Meier method was conducted using the log-rank test. The Cox proportional hazards regression model was used to analyze the independent predictor of mortality. The results with a $P$-value $<0.05$ were considered statistically significant.

\section{Results}

The baseline demographic and biochemical characteristics of all 466 patients are listed in Table 1 . Among the 466 patients enrolled in the study, the mean age was $72.98 \pm 10.12$ years old. 257 were male, 76 were of CKD 5 Stage and 390 were of CKD 3-4 Stage. The median HCAR was 0.25(0.08-0.65). 
Table 1

Baseline Characteristics of the Study Population based on HCAR Level

$\begin{array}{lllll}\text { Characteristics } & \text { Total } & \text { Lower HCAR } & \text { Higher HCAR }\end{array}$ P

Demographic

\begin{tabular}{|c|c|c|c|c|}
\hline Age(years) & $72.98 \pm 10.12$ & $73.60 \pm 9.17$ & $72.37 \pm 10.95$ & 0.191 \\
\hline Male, n (\%) & $257(55 \%)$ & $121(52 \%)$ & $136(58 \%)$ & 0.276 \\
\hline BMI $\left(\mathrm{kg} / \mathrm{m}^{2}\right)$ & $25.34 \pm 3.75$ & $25.21 \pm 3.57$ & $25.47 \pm 3.92$ & 0.458 \\
\hline $\mathrm{SBP}(\mathrm{mmHg})$ & $135.09 \pm 24.99$ & $137.50 \pm 24.54$ & $132.73 \pm 25.25$ & 0.039 \\
\hline DBP (mmHg) & $73.09 \pm 12.85$ & $74.02 \pm 12.80$ & $72.18 \pm 12.87$ & 0.122 \\
\hline \multicolumn{5}{|l|}{ Medical history } \\
\hline HT, n (\%) & $394(85 \%)$ & 198(86\%) & 196(83\%) & 0.365 \\
\hline $\mathrm{DM}, \mathrm{n}(\%)$ & $206(44 \%)$ & $107(47 \%)$ & $99(42 \%)$ & 0.32 \\
\hline Family history, n (\%) & $110(24 \%)$ & $56(24 \%)$ & $54(24 \%)$ & 0.709 \\
\hline Previous CAG, n (\%) & $116(24 \%)$ & $75(33 \%)$ & $38(16 \%)$ & $\begin{array}{l}<.001 \\
0.00\end{array}$ \\
\hline Stroke & $110(24 \%)$ & $55(24 \%)$ & $55(23 \%)$ & 0.877 \\
\hline Smoke, n (\%) & $224(48 \%)$ & $104(45 \%)$ & $120(51 \%)$ & 0.244 \\
\hline Alcohol drink, n (\%) & $113(24 \%)$ & $53(23 \%)$ & $81(34 \%)$ & 0.008 \\
\hline CKD 5 Stage, n (\%) & $76(16 \%)$ & $30(13 \%)$ & $46(19 \%)$ & 0.06 \\
\hline \multicolumn{5}{|l|}{ Laboratory test } \\
\hline $\begin{array}{l}\text { eGFR }(\mathrm{mL} / \mathrm{min} / 1.73 \\
\left.\mathrm{m}^{2}\right)\end{array}$ & $43.96 \pm 15.30$ & $45.51 \pm 14.90$ & $42.44 \pm 15.56$ & 0.03 \\
\hline $\mathrm{BUN}(\mu \mathrm{mol} / \mathrm{L})$ & $7.79(5.83-10.74)$ & $7.43(5.91-10.14)$ & $8.09(5.74-11.27)$ & 0.209 \\
\hline Serum K(mmol/L) & $4.27 \pm 0.48$ & $4.22 \pm 0.43$ & $4.32 \pm 0.52$ & 0.23 \\
\hline Serum $\mathrm{Na}(\mathrm{mmol} / \mathrm{L})$ & $139.48 \pm 6.84$ & $140.38 \pm 3.48$ & $138.63 \pm 8.88$ & 0.006 \\
\hline $\mathrm{TG}(\mathrm{mmol} / \mathrm{L})$ & $1.40(1.03-1.95)$ & $1.41(1.03-2.07)$ & $1.39(1.03-1.92)$ & 0.718 \\
\hline
\end{tabular}

DM, diabetes mellitus; FBG, fasting blood-glucose; HDL, high-density lipoprotein; HGB. Hemoglobin ;HT, hypertension; LDL, low-density lipoprotein; TC, total cholesterol; TG, triglyceride; UA, uric acid. eGFR, Estimated Glomerular Filtration Rate; CKD, chronic kidney disease; CAG, Corconary angiography; BUN, blood urea nitrogen; HbA1C, Hemoglobin A1C; WBC, WBC: white blood cell; ALT, alanine aminotransferase; AST, Aspertate Aminotransferase; CK-MB, Aspertate Aminotransferase; Tnl, troponin l; LVEF, left ventricular ejection fraction; HCAR, hs-CRP / albumin ratio. 


\begin{tabular}{|c|c|c|c|c|}
\hline \multirow[t]{2}{*}{ Characteristics } & Total & Lower HCAR & Higher HCAR & \multirow[t]{2}{*}{$\mathbf{P}$} \\
\hline & $(n=466)$ & $(n=230)$ & $(n=236)$ & \\
\hline $\mathrm{TC}(\mathrm{mmol} / \mathrm{L})$ & $4.28 \pm 1.07$ & $4.28 \pm 1.14$ & $4.29 \pm 1.00$ & 0.963 \\
\hline LDL-C(mmol/L) & $2.45 \pm 0.78$ & $2.44 \pm 0.82$ & $2.47 \pm 0.75$ & 0.713 \\
\hline $\mathrm{HDL}-\mathrm{C}(\mathrm{mmol} / \mathrm{L})$ & $1.03 \pm 0.24$ & $1.04 \pm 0.24$ & $1.02 \pm 0.24$ & 0.67 \\
\hline HbA1C(\%) & $6.30(5.78-7.30)$ & $6.30(5.80-7.20)$ & $6.30(5.75-7.45)$ & 0.789 \\
\hline $\mathrm{FBG}(\mathrm{mmol} / \mathrm{L})$ & $5.98(5.03-8.36)$ & $5.77(4.80-7.84)$ & $6.42(5.25-8.59)$ & $\begin{array}{l}< \\
0.001\end{array}$ \\
\hline WBC $\left(10^{9} / L\right)$ & $7.83(5.97-9.63)$ & $6.80(5.40-8.60)$ & $8.40(6.91-10.75)$ & $<.001$ \\
\hline $\mathrm{HBG}(\mathrm{g} / \mathrm{L})$ & $123.86 \pm 19.58$ & $124.42 \pm 19.62$ & $123.31 \pm 19.57$ & 0.539 \\
\hline PLT $\left(10^{9} / \mathrm{L}\right)$ & $215.47 \pm 72.12$ & $206.94 \pm 63.79$ & $223.78 \pm 78.65$ & 0.012 \\
\hline $\mathrm{UA}(\mu \mathrm{mol} / \mathrm{L})$ & $403.63 \pm 104.42$ & $397.06 \pm 92.78$ & $410.08 \pm 114.55$ & 0.188 \\
\hline $\operatorname{ALT}(\mathrm{U} / \mathrm{L})$ & $\begin{array}{l}18.00(12.00- \\
31.00)\end{array}$ & $\begin{array}{l}17.00(12.00- \\
26.00)\end{array}$ & $\begin{array}{l}20.00(13.00 \\
-40.50)\end{array}$ & 0.003 \\
\hline AST(U/L) & $\begin{array}{l}30.00(18.00- \\
71.00)\end{array}$ & $\begin{array}{l}24.00(17.00- \\
44.00)\end{array}$ & $\begin{array}{l}40.00(19.30 \\
-113.50)\end{array}$ & $<0.001$ \\
\hline CK-MB(U/L) & $4.00(1.80-15.05)$ & $3.10(1.60-7.70)$ & $5.60(2.20-25.25)$ & $<.001$ \\
\hline $\operatorname{Tnl}(\mathrm{ng} / \mathrm{ml})$ & $0.87(0.11-3.66)$ & $0.52(0.08-1.73)$ & $1.72(0.17-6.90)$ & $<0.001$ \\
\hline LVEF & $0.56 \pm 0.12$ & $0.57 \pm 0.11$ & $0.55 \pm 0.12$ & 0.073 \\
\hline NT-proBNP(pg/ml) & $\begin{array}{l}\text { 6066(1990- } \\
15840)\end{array}$ & $\begin{array}{l}3619(1406- \\
10400)\end{array}$ & $9611(2859-22277)$ & $<.001$ \\
\hline \multicolumn{5}{|c|}{ Drug use after admission } \\
\hline Antiplatelet, n (\%) & $436(94 \%)$ & $221(96 \%)$ & $215(91 \%)$ & 0.04 \\
\hline ACEI/ARB, n (\%) & $316(68 \%)$ & $160(70 \%)$ & $156(66 \%)$ & 0.503 \\
\hline ß-blocker, n (\%) & $344(74 \%)$ & 172(75\%) & 172(73\%) & 0.753 \\
\hline Statins, n (\%) & $410(88 \%)$ & 207(90\%) & 203(86\%) & 0.178 \\
\hline \multicolumn{5}{|c|}{$\begin{array}{l}\text { DM, diabetes mellitus; FBG, fasting blood-glucose; HDL, high-density lipoprotein; HGB. Hemoglobin } \\
\text {;HT, hypertension; LDL, low-density lipoprotein; TC, total cholesterol; TG, triglyceride; UA, uric acid. } \\
\text { eGFR, Estimated Glomerular Filtration Rate; CKD, chronic kidney disease; CAG, Corconary } \\
\text { angiography; BUN, blood urea nitrogen; HbA1C, Hemoglobin A1C; WBC, WBC: white blood cell; ALT, } \\
\text { alanine aminotransferase; AST, Aspertate Aminotransferase; CK-MB, Aspertate Aminotransferase; TnI, } \\
\text { troponin I; LVEF, left ventricular ejection fraction; HCAR, hs-CRP / albumin ratio. }\end{array}$} \\
\hline
\end{tabular}




\begin{tabular}{|c|c|c|c|c|}
\hline \multirow[t]{2}{*}{ Characteristics } & Total & Lower HCAR & Higher HCAR & \multirow[t]{2}{*}{$\mathbf{P}$} \\
\hline & $(n=466)$ & $(n=230)$ & $(n=236)$ & \\
\hline 2 year mortality, n (\%) & $68(15 \%)$ & $23(10 \%)$ & $45(19 \%)$ & 0.006 \\
\hline \multicolumn{5}{|c|}{$\begin{array}{l}\text { DM, diabetes mellitus; FBG, fasting blood-glucose; HDL, high-density lipoprotein; HGB. Hemoglobin } \\
\text {;HT, hypertension; LDL, low-density lipoprotein; TC, total cholesterol; TG, triglyceride; UA, uric acid. } \\
\text { eGFR, Estimated Glomerular Filtration Rate; CKD, chronic kidney disease; CAG, Corconary } \\
\text { angiography; BUN, blood urea nitrogen; HbA1C, Hemoglobin A1C; WBC, WBC: white blood cell; ALT, } \\
\text { alanine aminotransferase; AST, Aspertate Aminotransferase; CK-MB, Aspertate Aminotransferase; Tnl, } \\
\text { troponin l; LVEF, left ventricular ejection fraction; HCAR, hs-CRP / albumin ratio. }\end{array}$} \\
\hline
\end{tabular}

The scatter diagrams and correlation analysis showed the correlation between HCAR and the indices reflecting the cardiac function, the severity of myocardial damage and renal function: NT-proBNP, LVEF, CK-MB, Tnl and eGFR (Fig. 2). Besides, the HCAR was significantly correlated with WBC $(r=0.331, P<$ $0.001)$, hs-CRP $(r=0.996, P<0.001)$ and ALT $(r=0.139, P=0.003)$, which indicated that MLR could also reflect both the degree of inflammation and hepatic function.

The ROC analysis was used to analyze HCAR efficiency in predicting 2-year mortality. A cut-off value of 0.24 of HCAR showed the best efficiency, with a sensitivity of $66.18 \%$ and specificity of $52.21 \%$ (ROC area under the curve: $0.60,95 \% \mathrm{Cl}: 0.53-0.68, P<0.001$; Fig. $3 \mathrm{~A}$ ). The ROC curve was also used to assess HCAR efficiency in predicting the stage of CKD (Stage 3-4 vs. Stage 5), and a cut-off value of 0.15 had a sensitivity of $77.63 \%$ and specificity of $39.74 \%$ (ROC area under the curve: $0.61,95 \% \mathrm{Cl}: 0.54-0.68, P=$ 0.003; Fig. 3B).

The patients were divided into 2 groups according to the HCAR cutoff value of 0.24 in predicting 2-year mortality. The group with a higher HCAR (HCAR $\leq 0.24)$ included 236 patients ( 136 males, aged $72.37 \pm$ 10.95 years old) and the group with a lower HCAR (HCAR < 0.24$)$ included 230 patients $(121$ males, aged $73.60 \pm 9.17$ years old). Overall, the group with a higher HCAR had lower eGFR and higher FBG, WBC, PLT, ALT, AST, CK-MB, Tnl and NT-proBNP $(P<0.05)$ (Table 1). Besides, the group with a higher HCAR was significantly associated with a higher 2-year mortality (45/236 (19\%) vs. 23/230 (10\%), $P=0.006)$ (Fig. 4). The Kaplan-Meier curve showed that the group with a higher HCAR had a worse prognosis (logrank $P<0.001$, Fig. 5).

As shown in Table 2, compared to survivors, nonsurvivors had lower BMI, eGFR, HBG, and LVEF and older age, AST, NT-proBNP and HCAR $(P<0.05)$. CK-MB tended to be higher in nonsurvivors with a critical $P$ value of 0.095 . The use of antiplatelet agents, ACEI/ARB, $\beta$-blocker and statins could reduce mortality significantly $(P<0.01)$. Finally, a Cox regression analysis was used to assess the risk factors for 2-year mortality. Based on the results shown in Table 2, traditional risk factors, including age, gender, CKD stage, $B M I, H T, D M$, family history of CAD, TG ,TC, LDL-C, HDL-C, smoke and FBG, LVEF, NT-proBNP, HCAR, the use of antiplatelet agents, ACEI/ARB, $\beta$-blocker and statins were included into the multivariable Cox regression analysis. The regression analysis shown in Table 3 demonstrated that HCAR was an 
independent risk factor for 2-year mortality (OR: 1.779, 95\% Cl: 1.017-3.112, $\mathrm{P}=0.043$ ), together with NTproBNP, age and the use of antiplatelet agent and statins. 
Table 2

Characteristics of the Study Population based on all cause morality

\begin{tabular}{|c|c|c|c|}
\hline \multirow[t]{2}{*}{ Characteristics } & Survivors & Nonsurvivors & \multirow[t]{2}{*}{$\mathbf{P}$} \\
\hline & $(n=398)$ & $(n=68)$ & \\
\hline \multicolumn{4}{|l|}{ Demographic } \\
\hline Age(years) & $72.36 \pm 10.05$ & $76.59 \pm 9.85$ & 0.001 \\
\hline Male, n (\%) & $223(56 \%)$ & $34(50 \%)$ & 0.355 \\
\hline $\mathrm{BMI}\left(\mathrm{kg} / \mathrm{m}^{2}\right)$ & $25.53 \pm 3.61$ & $24.24 \pm 4.34$ & 0.022 \\
\hline SBP $(\mathrm{mmHg})$ & $135.66 \pm 24.92$ & $131.71 \pm 25.28$ & 0.228 \\
\hline DBP (mmHg) & $73.41 \pm 12.93$ & $71.21 \pm 12.28$ & 0.192 \\
\hline \multicolumn{4}{|l|}{ Medical history } \\
\hline HT, n (\%) & $333(84 \%)$ & $61(90 \%)$ & 0.203 \\
\hline DM, n (\%) & $176(44 \%)$ & $30(44 \%)$ & 0.987 \\
\hline CAD family history & $95(24 \%)$ & $15(22 \%)$ & 0.745 \\
\hline Previous CAG, n (\%) & $100(25 \%)$ & 13(19\%) & 0.285 \\
\hline Stroke, n (\%) & $92(23 \%)$ & $18(26 \%)$ & 0.547 \\
\hline Smoke, n (\%) & $190(48 \%)$ & $34(50 \%)$ & 0.73 \\
\hline Alcohol drink, n (\%) & $116(29 \%)$ & $18(26 \%)$ & 0.644 \\
\hline CKD 5 Stage, n (\%) & $58(15 \%)$ & $18(26 \%)$ & 0.014 \\
\hline \multicolumn{4}{|l|}{ Laboratory test } \\
\hline $\mathrm{eGFR}\left(\mathrm{mL} / \mathrm{min} / 1.73 \mathrm{~m}^{2}\right)$ & $44.88 \pm 15.09$ & $38.56 \pm 15.52$ & 0.002 \\
\hline BUN ( $\mu \mathrm{mol} / \mathrm{L})$ & $7.57(5.83-10.89)$ & $8.67(5.62-11.51)$ & 0.231 \\
\hline Serum K(mmol/L) & $4.26 \pm 0.47$ & $4.33 \pm 0.54$ & 0.27 \\
\hline Serum $\mathrm{Na}(\mathrm{mmol} / \mathrm{L})$ & $139.53 \pm 7.24$ & $139.20 \pm 3.87$ & 0.713 \\
\hline $\mathrm{TG}(\mathrm{mmol} / \mathrm{L})$ & $1.43(1.02-1.99)$ & $1.33(1.03-1.82)$ & 0.417 \\
\hline TC(mmol/L) & $4.31 \pm 1.09$ & $4.13 \pm 0.94$ & 0.211 \\
\hline LDL-C(mmol/L) & $2.47 \pm 0.80$ & $2.37 \pm 0.70$ & 0.357 \\
\hline HDL-C(mmol/L) & $1.03 \pm 0.24$ & $1.04 \pm 0.25$ & 0.681 \\
\hline HbA1C(\%) & $6.30(5.70-7.30)$ & $6.30(5.80-7.30)$ & 0.675 \\
\hline
\end{tabular}




\begin{tabular}{|c|c|c|c|}
\hline \multirow[t]{2}{*}{ Characteristics } & Survivors & Nonsurvivors & \multirow[t]{2}{*}{$\mathbf{P}$} \\
\hline & $(n=398)$ & $(n=68)$ & \\
\hline $\mathrm{FBG}(\mathrm{mmol} / \mathrm{L})$ & $5.94(5.00-8.21)$ & $6.87(5.08-9.21)$ & 0.111 \\
\hline WBC $\left(10^{9} / \mathrm{L}\right)$ & $7.83(6.10-9.56)$ & 7.91(5.54-10.68) & 0.96 \\
\hline HBG (g/L) & $124.61 \pm 19.12$ & $119.46 \pm 21.72$ & 0.045 \\
\hline $\operatorname{PLT}\left(10^{9} / \mathrm{L}\right)$ & $216.47 \pm 72.66$ & $209.56 \pm 69.09$ & 0.465 \\
\hline $\mathrm{UA}(\mu \mathrm{mol} / \mathrm{L})$ & $400.90 \pm 103.90$ & $419.36 \pm 106.83$ & 0.185 \\
\hline ALT(U/L) & $18.00(12.00-31.00)$ & $22.00(12.00-47.50)$ & 0.132 \\
\hline AST(U/L) & $29.00(18.00-67.00)$ & $39.40(20.00-146.00)$ & 0.049 \\
\hline CK-MB(U/L) & $4.00(1.70-13.90)$ & $4.30(2.50-21.15)$ & 0.095 \\
\hline Tnl(ng/ml) & $0.81(0.10-3.45)$ & $1.73(0.17-3.96)$ & 0.168 \\
\hline LVEF & $0.57 \pm 0.11$ & $0.52 \pm 0.12$ & $<0.001$ \\
\hline NT-proBNP(pg/ml) & 5316(1834-13030) & $20433(5318-30000)$ & $<0.001$ \\
\hline Higher HCAR & $191(48 \%)$ & $45(66 \%)$ & 0.006 \\
\hline \multicolumn{4}{|c|}{ Drug use after admission } \\
\hline Antiplatelet, n (\%) & $387(97 \%)$ & $49(72 \%)$ & $<0.001$ \\
\hline ACEI /ARB, n (\%) & $279(70 \%)$ & $37(54 \%)$ & 0.009 \\
\hline$\beta$-blocker, n (\%) & $305(77 \%)$ & $39(57 \%)$ & 0.001 \\
\hline Statins, n (\%) & $367(92 \%)$ & $43(63 \%)$ & $<0.001$ \\
\hline
\end{tabular}

Table 3

Cox regression analysis in relation to 2-year mortality

\begin{tabular}{|llllll|}
\hline Variable & B & Wald & P Value & OR & 95\% Cl \\
\hline Age & 0.046 & 10.465 & 0.001 & 1.048 & $1.018-1.077$ \\
\hline Antiplatelet & -1.398 & 7.701 & 0.006 & 0.247 & $0.092-0.663$ \\
\hline Statins & -0.943 & 4.520 & 0.033 & 0.390 & $0.163-0.929$ \\
\hline NT-proBNP & $<0.001$ & 16.380 & $<0.001$ & 1.00005 & $1.00003-1.00008$ \\
\hline Higher HCAR & 0.576 & 4.077 & 0.043 & 1.779 & $1.017-3.112$ \\
\hline
\end{tabular}

\section{Discussion}


The key role of inflammation in diseases development has attracted more attention, and the studies on inflammatory indices have been highlighted in recent years. At present, hs-CRP and CRP are widely used as indicators of inflammation. Although both the two indices are actually measured with C-reactive protein, the clinical significance of CRP and hs-CRP is not exactly the same[17, 18]. CRP has good performance in the diagnosis of infectious and connective tissue diseases, whereas, hs-CRP, due to its higher sensitivity, precision and reproducibility, seems better for diagnosis of cardio-and cerebrovascular disease[14, 18-20]. Contrary to other specific inflammatory factors or markers, the hs-CRP and serum albumin are easily measured in clinics, and are widely used in many primary hospitals, with a high penetration rate, stable results and easy to observation. Through the combination of the two indicators above, HCAR has the advantages of simplicity, low-cost and high reliability. On the basis of making full use of the existing biochemical examination, HCAR can help to excavate more detailed diagnosis and treatment information, provide reference for clinical diagnosis and treatment, and effectively save costs, which is potentially valuable in clinical application.

Previous studies have suggested that coronary atherosclerosis is a multistep and chronic inflammatory process. Inflammation plays a key role in the formation, development and rupture of atherosclerotic plaques. In 2019, Zhuang conducted a randomized clinical trial, enrolling 3802 subjects followed up for 5.01 years, and it was found that hs-CRP was positively associated with the incidence of coronary heart disease in the general population[16]. In 2014, Daniel's study identified hs-CRP as an independent predictive factor of 30-day mortality in STEMI patients[21]. Serum albumin level can reflect the nutritional status of patients and predict prognosis of cancer, infection and critical patients. A low albumin level indicates poor prognosis of patients. Therefore, by combining hs-CRP and albumin, the predictive value of HCAR has gained increasing attention of researchers. Wang et al. have shown that HCAR is closely related to the incidence of short-term adverse cardiovascular events in patients with acute coronary syndrome, and could provide help to risk stratification[22]. However, the clinical value of HCAR in AMI patients has not been reported, especially in patients with chronic kidney disease.

Using scatter diagrams and correlation analysis, this study showed that HCAR was significantly and positively correlated with inflammatory indices, leukocyte count and hs-CRP, in AMI patients with CKD undergoing CAG. Thus, HCAR is a useful indicator closely related to hs-CRP, which could also reflect inflammation. Besides, HCAR was positively correlated with NT proBNP, CK MB, Tnl, AST and ALT, and negatively correlated with LVEF and eGFR $(P<0.05)$. These findings indicate that HCAR is significantly associated with cardiac, renal and hepatic functions. Thus, in patients with AMI combined with CKD, HCAR may reflect the severity of myocardial infarction, as well as cardiac and renal function.

The current study also showed that in AMI patients with CKD undergoing CAG, HCAR has a good value in predicting mortality. The diagnostic efficiency is the highest when HCAR is 0.24 , with a sensitivity of $66.2 \%$ and specificity of $52.0 \%$. Therefore, in this study, the patients were divided into two groups with the HCAR value of 0.24 as the cut-off value. Consistent with the above correlation analysis, the group with a higher HCAR was negatively correlated with eGFR, and positively correlated with NT-proBNP, CK MB, Tnl, ALT and AST, which further confirmed that HCAR has certain advantages in reflecting the severity of 
myocardial infarction, as well as cardiac and renal function. Especially, a negative correlation between HCAR level and mortality was observed, suggesting that HCAR may predict prognosis of the patients. Further analysis of survival rates of patients showed that the survival time of patients in the group with a lower HCAR was significantly longer than that in the group with a higher HCAR. Hence, a comprehensive analysis of mortality risk factors was conducted. The Cox survival analysis indicated that higher HCAR level, older age and higher NT-proBNP were independent predictors of 2-year mortality of AMI patients with CKD undergoing CAG.

Also, there was a negative correlation between HCAR and eGFR $(P<0.05)$. The predictive value of HCAR in CKD patients was also investigated. The results showed that HCAR had a good diagnostic value in predicting the severity of CKD. HCAR was most efficient in the diagnosis of CKD staging when HCAR was 0.17 , with a sensitivity of $77.6 \%$ and specificity of $39.7 \%$. These results indicate that HCAR is clinically valuable in evaluating the renal function of AMI patients with CKD undergoing CAG.

However, this study also has some limitations. Firstly, this was a retrospective and single-center study with a limited sample size. Thus, a prospective, multicenter and randomized controlled trial is required to corroborate our findings. Secondly, this study only focused on value of HCAR in predicting the 2-year mortality of AMI patients with CKD undergoing CAG. Further studies need to be conducted on MACE, CKD progression and mortality in 5years or even longer time. Thirdly, this study only evaluated the association of HCAR level with prognosis of the patients upon admission, lacking the data reflecting changes over time. In the future, more information should be collected to gain more insight into the diagnostic value of HCAR.

In conclusion, this study indicates that HCAR may comprehensively reflect the condition of AMI patients with CKD undergoing CAG. HCAR could not only reflect the degree of inflammation, but also predict the severity of myocardial infarction, renal function, liver function and prognosis. With its advantages of simplicity, cost-effectiveness, and reliability, HCAR is potentially applied clinically in the future.

\section{Abbreviations}

AMI: Acute myocardial infarction; CKD: Chronic kidney disease; Hs-CRP: High sensitivity C-reactive protein; Alb: albumin; HCAR: High sensitivity C-reactive protein to albumin ratio; CAG: Coronary angiography; ROC: Receiver operating characteristic; PCl: Percutaneous coronary intervention (PCI); eGFR: Estimated glomerular filtration rate; LVEF: Left ventricular ejection fraction

\section{Declarations}

\section{Acknowledgements}

We thank sincerely all patients and staff contributing to this study.

\section{Authors' contributions}


Bo Zuo and Jiayu Li were devoted to the analysis and interpretation of the data and writing for this article. Hui Chen designed this research. All authors reviewed and approved the manuscript.

\section{Funding}

This study was supported by the National Natural Science Foundation of China (No. 82000328).

\section{Availability of data and materials}

The datasets used and/or analysed during the current study are available from the corresponding author on reasonable request.

\section{Ethics approval and consent to participate}

This study was approved by the ethics committee of Beijing Friendship Hospital, Capital Medical University and written informed consent was obtained from all patients

Consent for publication

Not applicable.

\section{Competing interests}

The authors declare that they have no competing interests.

\section{Author details}

1 Department of Cardiology, Cardiovascular Centre, Beijing Friendship Hospital, Capital Medical University, NO. 52 Yongan Road, Beijing; 100052, China.

\section{References}

1. Falsoleiman H, Fatehi GH, Dehghani M, Shakeri MT, Bayani B, Ahmadi M, Rohani A: Clinical outcome, and survival between primary percutaneous coronary intervention versus fibrinolysis in patients older than 60 years with acute myocardial infarction. Heart Views 2012, 13(4):129-131.

2. Nyman U, Bjork J, Aspelin P, Marenzi G: Contrast medium dose-to-GFR ratio: a measure of systemic exposure to predict contrast-induced nephropathy after percutaneous coronary intervention. Acta Radiol 2008, 49(6):658-667.

3. Sarnak MJ, Amann K, Bangalore S, Cavalcante JL, Charytan DM, Craig JC, Gill JS, Hlatky MA, Jardine AG, Landmesser $U$ et al: Chronic Kidney Disease and Coronary Artery Disease: JACC State-of-the-Art Review. J Am Coll Cardiol 2019, 74(14):1823-1838.

4. Newsome P, Francque S, Harrison S, Ratziu V, Van Gaal L, Calanna S, Hansen M, Linder M, Sanyal A: Effect of semaglutide on liver enzymes and markers of inflammation in subjects with type 2 diabetes and/or obesity. Aliment Pharmacol Ther 2019, 50(2):193-203. 
5. Kubo S, Kitamura A, Imano H, Cui R, Yamagishi K, Umesawa M, Muraki I, Kiyama M, Okada T, Iso H: Serum Albumin and High-Sensitivity C-reactive Protein are Independent Risk Factors of Chronic Kidney Disease in Middle-Aged Japanese Individuals: the Circulatory Risk in Communities Study. $J$ Atheroscler Thromb 2016, 23(9):1089-1098.

6. Wang J, Tang B, Liu X, Wu X, Wang H, Xu D, Guo Y: Increased monomeric CRP levels in acute myocardial infarction: a possible new and specific biomarker for diagnosis and severity assessment of disease. Atherosclerosis 2015, 239(2):343-349.

7. Calik AN, Inan D, Karatas MB, Akdeniz E, Genc D, Canga Y, Cinar T, Emre A: The association of preprocedural C-reactive protein/albumin ratio with in-stent restenosis in patients undergoing iliac artery stenting. J Cardiovasc Thorac Res 2020, 12(3):179-184.

8. Oduncu V, Erkol A, Karabay CY, Kurt M, Akgun T, Bulut M, Pala S, Kirma C: The prognostic value of serum albumin levels on admission in patients with acute ST-segment elevation myocardial infarction undergoing a primary percutaneous coronary intervention. Coron Artery Dis 2013, 24(2):8894.

9. Bergstrom J: Nutrition and mortality in hemodialysis. J Am Soc Nephrol 1995, 6(5):1329-1341.

10. Duman H, Cinier G, Bakirci EM, Simsek Z, Hamur H, Degirmenci H, Emlek N: Relationship Between CReactive Protein to Albumin Ratio and Thrombus Burden in Patients With Acute Coronary Syndrome. Clin Appl Thromb Hemost 2019, 25:1076029618824418.

11. Karabag Y, Cagdas M, Rencuzogullari I, Karakoyun S, Artac I, llis D, Yesin M, Ciftci H, Erdogdu HI, Tanboga IH: The C-Reactive Protein to Albumin Ratio Predicts Acute Kidney Injury in Patients With ST-Segment Elevation Myocardial Infarction Undergoing Primary Percutaneous Coronary Intervention. Heart Lung Circ 2019, 28(11):1638-1645.

12. He S, Wang Y, Chen H, Yang L, Liang S, Lu L, Chen Y: C-Reactive Protein/Albumin Ratio (CAR) as a Prognostic Factor in Patients with Non-Metastatic Nasopharyngeal Carcinoma. J Cancer 2016, 7(15):2360-2366.

13. Oh TK, Ji E, Na HS, Min B, Jeon YT, Do SH, Song IA, Park HP, Hwang JW: C-Reactive Protein to Albumin Ratio Predicts 30-Day and 1-Year Mortality in Postoperative Patients after Admission to the Intensive Care Unit. J Clin Med 2018, 7(3).

14. Xu H, Hu L, Wei X, Niu J, Gao Y, He J, Hou J: The Predictive Value of Preoperative High-Sensitive CReactive Protein/Albumin Ratio in Systemic Inflammatory Response Syndrome After Percutaneous Nephrolithotomy. J Endourol 2019, 33(1):1-8.

15. Oh TK, Choi YR, Cho JY, Yoon YS, Han HS, Park IS, Ryu JH: The High-Sensitivity C-Reactive Protein/Albumin Ratio Predicts Long-Term Oncologic Outcomes after Curative Resection for Hepatocellular Carcinoma. J Clin Med 2018, 7(6).

16. Zhuang Q, Shen C, Chen Y, Zhao X, Wei P, Sun J, Ji Y, Chen X, Yang S: Association of high sensitive Creactive protein with coronary heart disease: a Mendelian randomization study. BMC Med Genet 2019, 20(1):170. 
17. Moutachakkir M, Lamrani Hanchi A, Baraou A, Boukhira A, Chellak S: Immunoanalytical characteristics of C-reactive protein and high sensitivity C-reactive protein. Ann Biol Clin (Paris) 2017, 75(2):225-229.

18. Pathak A, Agrawal A: Evolution of C-Reactive Protein. Front Immuno/ 2019, 10:943.

19. Castro AR, Silva SO, Soares SC: The Use of High Sensitivity C-Reactive Protein in Cardiovascular Disease Detection. J Pharm Pharm Sci 2018, 21(1):496-503.

20. Zhou Y, Han W, Gong D, Man C, Fan Y: Hs-CRP in stroke: A meta-analysis. Clin Chim Acta 2016, 453:21-27.

21. Ribeiro DR, Ramos AM, Vieira PL, Menti E, Bordin OL, Jr., Souza PA, Quadros AS, Portal VL: Highsensitivity C-reactive protein as a predictor of cardiovascular events after ST-elevation myocardial infarction. Arq Bras Cardiol 2014, 103(1):69-75.

22. Wang W, Ren D, Wang CS, Li T, Yao HC, Ma SJ: Prognostic efficacy of high-sensitivity C-reactive protein to albumin ratio in patients with acute coronary syndrome. Biomark Med 2019, 13(10):811820.

\section{Figures}




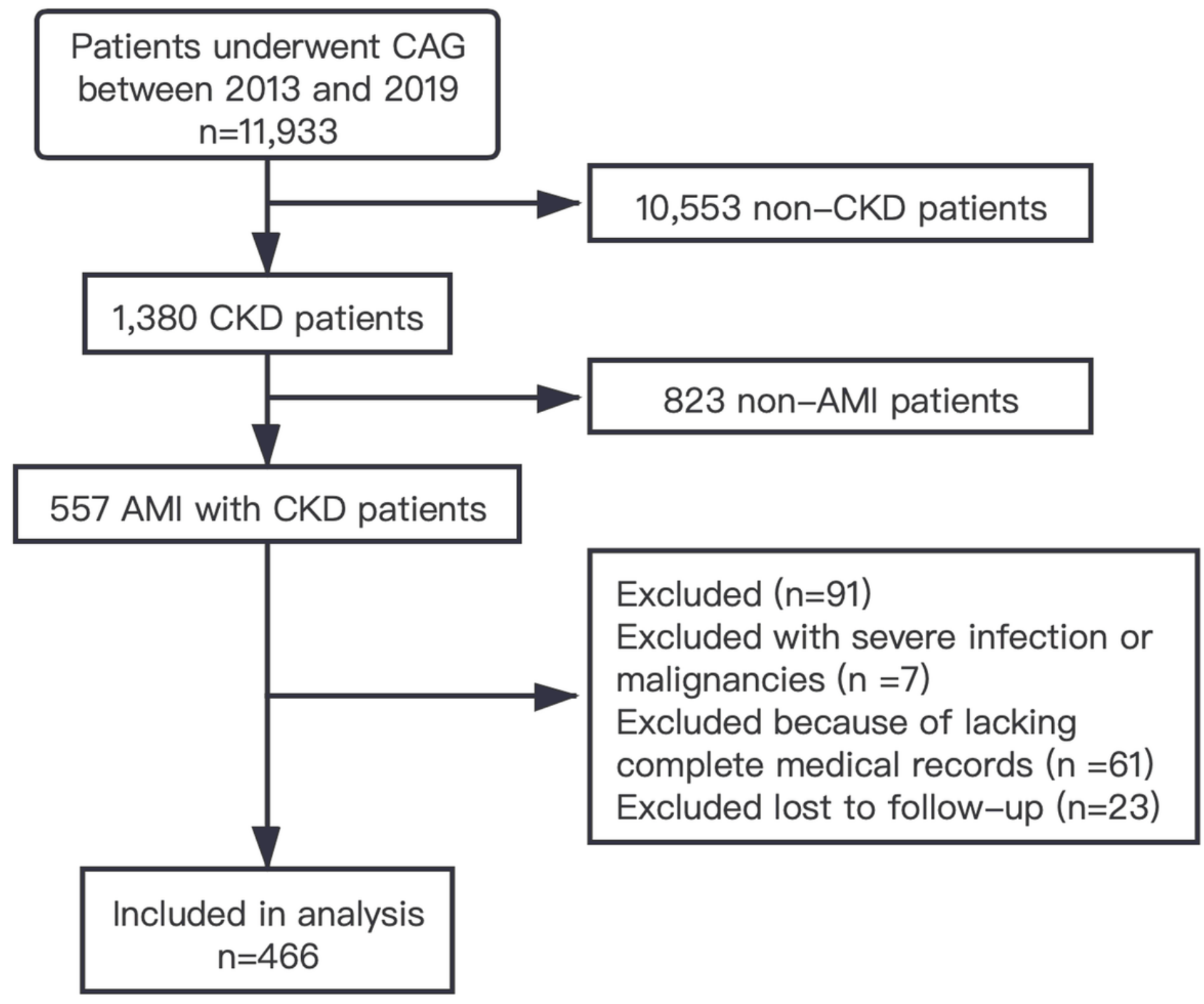

Figure 1

Flow chart of patient selection. AMI, acute myocardial infarction; CKD, chronic kidney disease; CAG, corconary angiography. 
A

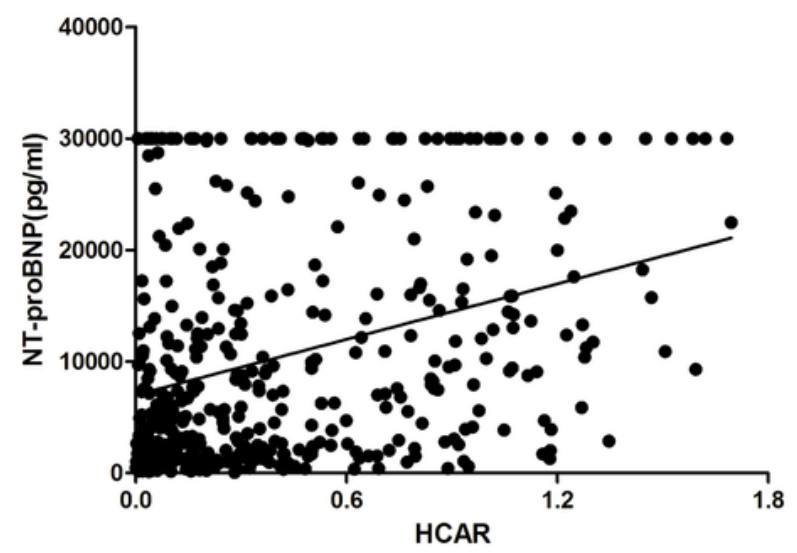

C

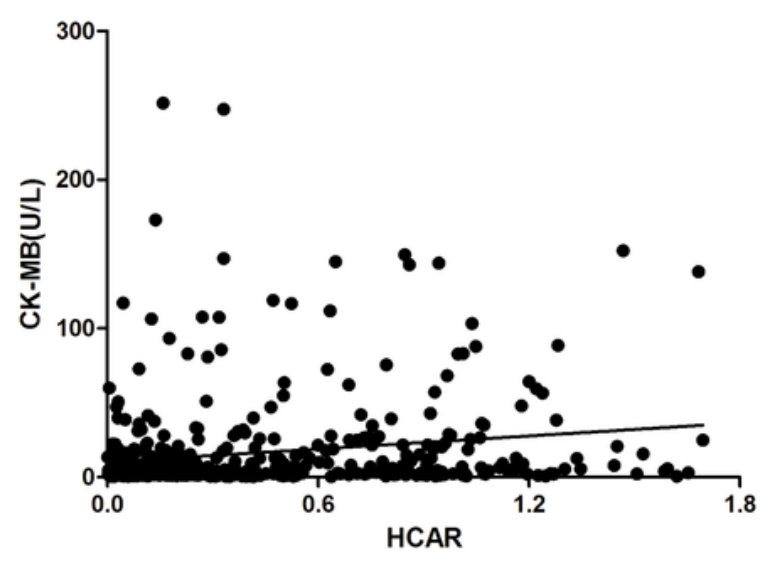

$\mathbf{E}$

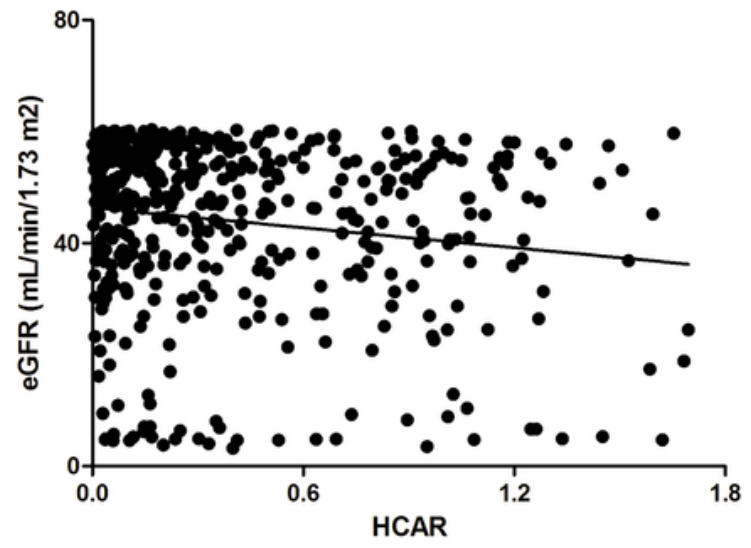

B

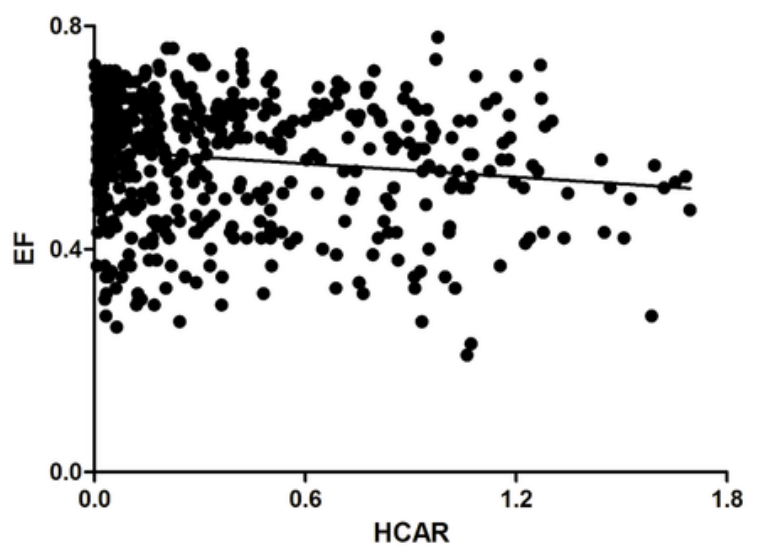

D

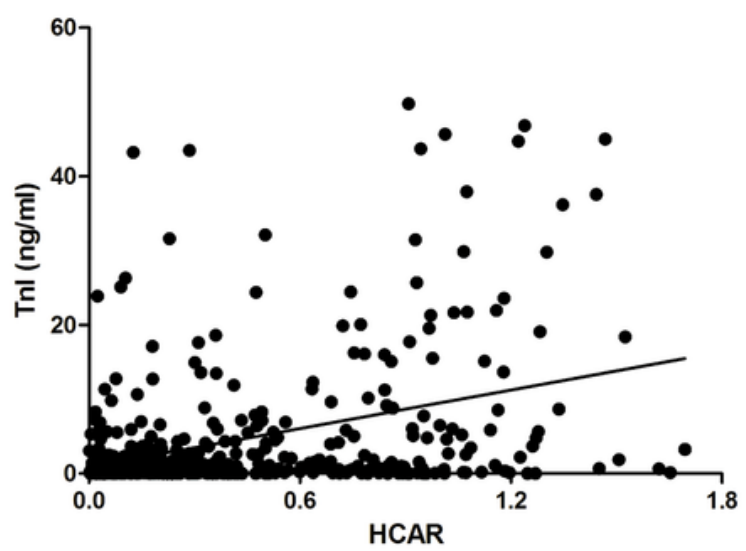

$\mathbf{F}$

\begin{tabular}{ccc}
\hline Variable & $\mathrm{r}$ & $\mathrm{P}$ \\
\hline NT-proBNP & 0.346 & $<0.001$ \\
LVEF & -0.143 & 0.002 \\
CK-MB & 0.242 & $<0.001$ \\
TnI & 0.303 & $<0.001$ \\
eGFR & -0.155 & $<0.001$ \\
\hline
\end{tabular}

Figure 2

The scatter diagrams and correlation analysis of HCAR. A: The scatter diagram of HCAR and NT-proBNP. B: The scatter diagram of HCAR and EF. C: The scatter diagram of HCAR and CK-MB.D: The scatter diagram of HCAR and Tnl. E: The scatter diagram of HCAR and eGFR. F: The correlation analysis of HCAR. 
A

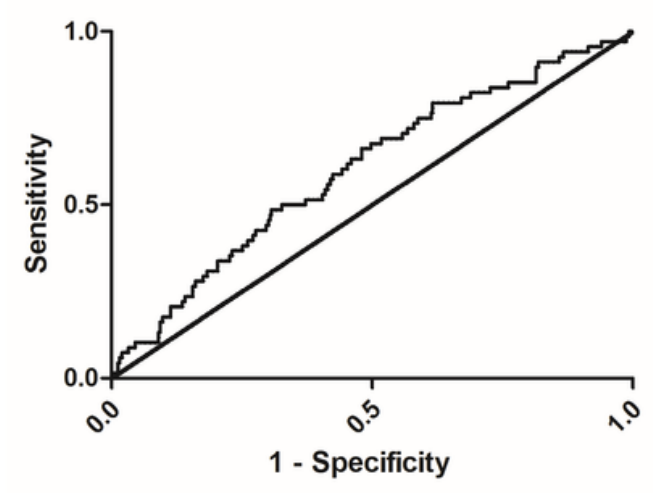

B

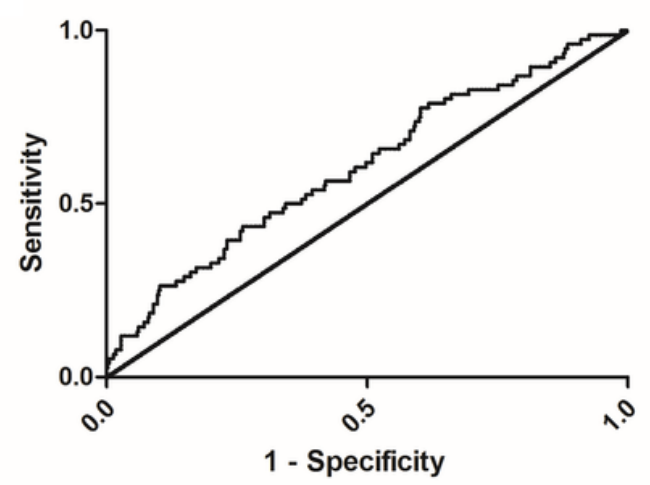

Figure 3

ROC curve of HCAR in predicting 2-year mortality and the stage of CKD. A: ROC curve of HCAR in predicting 2-year mortality. B: ROC curve of HCAR in predicting the stage of CKD. 


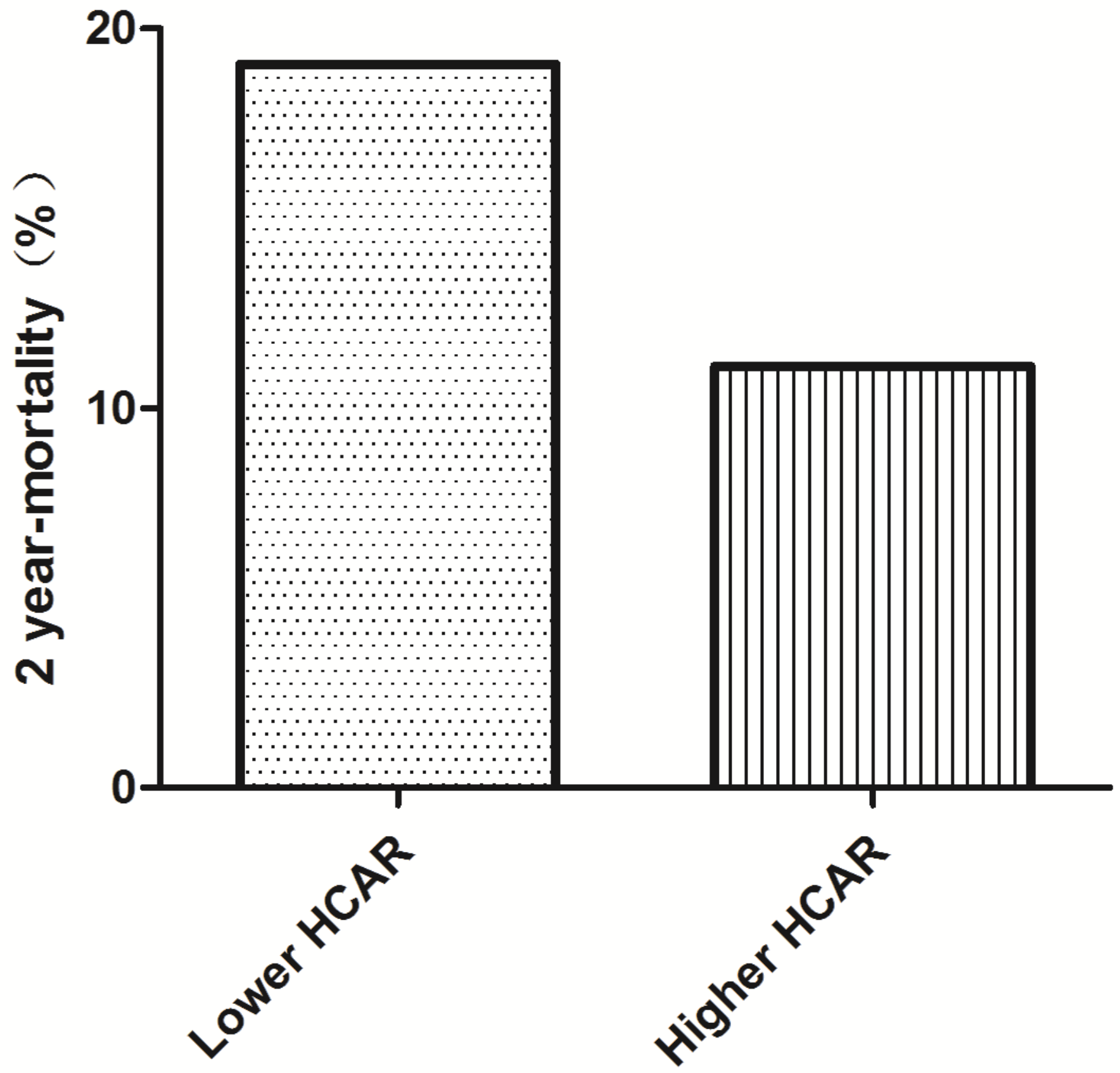

Figure 4

The comparison of 2-year mortality based on HCAR level. 


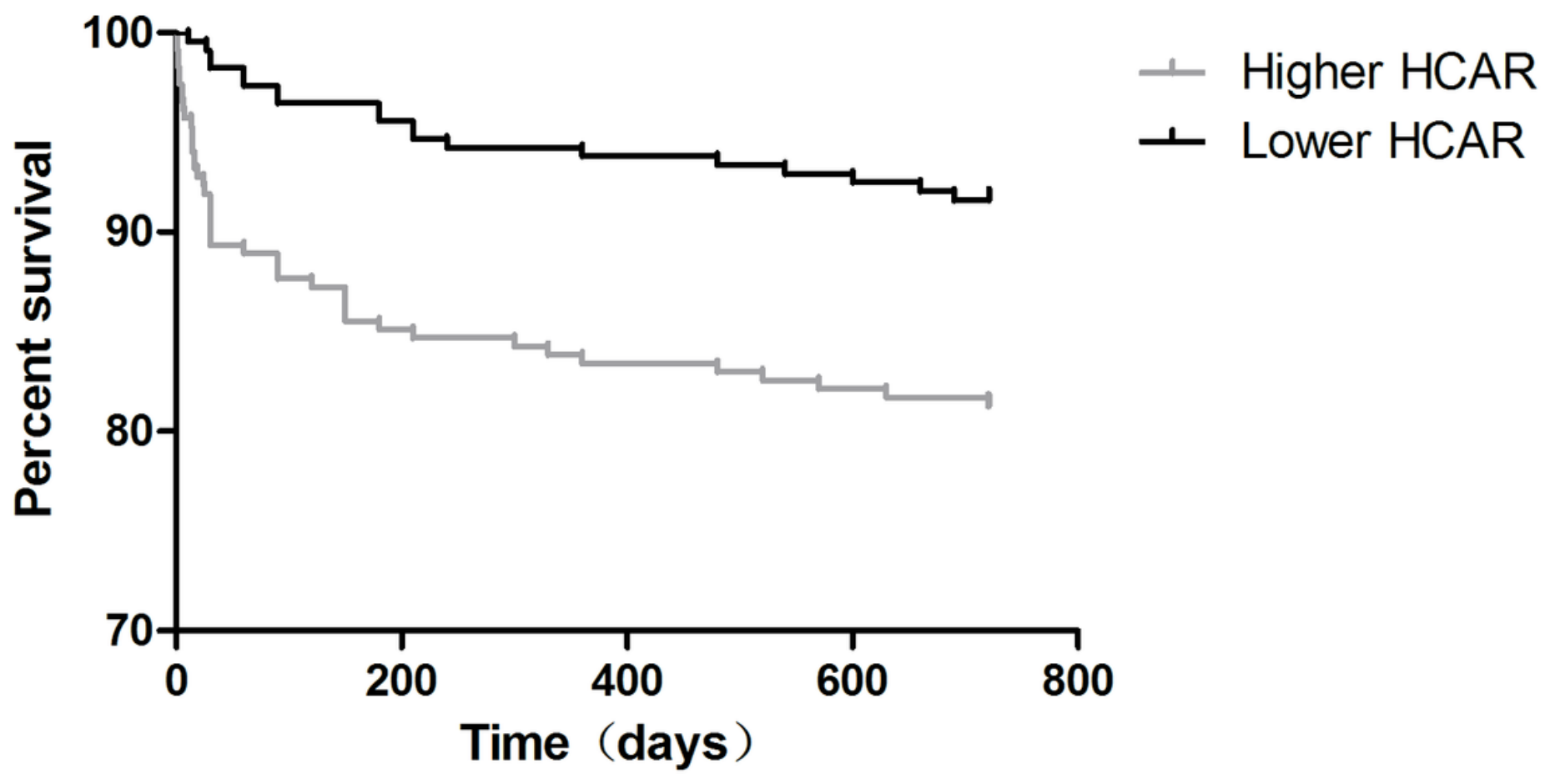

Figure 5

The Kaplan-Meier curve analysis of 2-year mortality based on the HCAR level. 